\title{
Capability-based Planning with ArchiMate Linking Motivation to Implementation
}

\author{
Adina Aldea ${ }^{1,2}$, Maria Eugenia Iacob ${ }^{1}$ and Jos Van Hillegersberg ${ }^{1}$, Dick Quartel ${ }^{2}$ and Henry Franken ${ }^{2}$ \\ ${ }^{I}$ Centre for Telematics and Information Technology, University of Twente, Enschede, The Netherlands \\ ${ }^{2}$ BiZZdesign, Enschede, The Netherlands \\ \{a.i.aldea,m.e.iacob,j.vanhillegersberg\}@utwente.nl, \{a.aldea,d.quartel,h.franken\}@bizzdesign.nl
}

Keywords: Capability-based Planning, ArchiMate, Enterprise Architecture, Strategy Planning, Business IT Alignment.

\begin{abstract}
This paper proposes a methodology for capability-based planning (CBP) and investigates how it can be modelled with ArchiMate. This can be considered an important step in aligning Business and IT. By having a common language to express organisational plans, enterprise architects can engage business leaders to plan organisational change based on business outcomes, rather than projects, processes and applications. This is possible because CBP is centred on realising strategic goals by focusing on what an organisation can do, rather than how it can do it. In order to determine a methodology for CBP we look at current research and practice, and propose a generic set of steps. Based on this, we analyse the ArchiMate 2.1 Specification for suitability and propose the addition of the Capability and Metric concepts. In the last section we validate our proposed methodology and metamodel with the help of a case study.
\end{abstract}

\section{INTRODUCTION}

In today's dynamic environment, organisations need to be ready and able to plan and implement change at a quicker pace. Organisations that rely on previous success and persist with strategies that have worked in the past have shown a decline in performance in situations of radical environment change (Audia et al., 2000).

Strategic management research has investigated for the past few decades how organisations can gain and maintain competitive advantage in such dynamic situations. This has led to the formulation of multiple theories, with a focus on Resources (Barney, 1991) and Capabilities ((Teece and Pisano, 1994), (Eisenhardt and Martin, 2000)) as a source of competitive advantage. Since the resource-based theories have been criticised to have major limitations (Kraaijenbrink et al., 2010), the focus has shifted to capability-based theories as a source for competitive advantage.

One of the most recent large-scale applications of capability-based theories can be seen in the defence sector. In the past decade, CBP has become a standard in defence planning throughout the NATO alliance (De Spiegeleire, 2011) and has been widely adopted by the Defence community (TTCP) (Hales and Chouinard, 2011).
The need for CBP in the context of organisations has become more apparent in the recent years. More and more researches identify capabilities as the way to link business and IT ((Danesh and Yu, 2014), (Stirna et al., 2012)), to link business outcomes to IT (Miklos, 2012), and all-in-all as a solution for improving the business and IT alignment ((Lee and Song, 2011), (Zdravkovic et al., 2013)).

Given this increased interest in capabilities, both in theory and in practice, it is no surprise that this concept has recently surfaced in enterprise architecture (EA). EA frameworks such as TOGAF (Open Group, 2011), have already introduced basic notions of CBP and its role in designing, planning and implementing organisational change. An approach to integrate resources and capabilities in ArchiMate, a modelling language for EA which is often used with TOGAF, has already been proposed by Iacob et al. (2012). One of the main issues addressed by CBP in this context is providing architects and business people with a common ground to initiate discussions in terms of business outcomes (increased output and quality, lower costs, revenue growth, improved market share) instead of projects, processes and applications (Scott, 2009).

Even though CBP is already being used with EA frameworks, there is little research into how to design, assess, implement and monitor capabilities, 
and also how to use this in a real case (Miklos, 2012). Preliminary research presented by Papazoglou (2014) describes a capability-based methodology that can be used in collaboration with TOGAF and modelled with ArchiMate. Although this research presents interesting ideas it still has several limitations regarding the possibility of making iterations, the large number of assumptions made and the validation of the method which was done by using a fictitious case study.

The goal and contribution of this paper is to propose a methodology for CBP that can be used independently of other domains such as strategic management or EA, but also in collaboration with these domains. Furthermore we investigate if and how the proposed CBP methodology can be modelled with ArchiMate. The ArchiMate modelling language was chosen as a basis for this work because it already contains a series of strategic concepts, an extension for the concepts of Capability and Resource has already been proposed by Iacob et al. (2012) and Azevedo et al. (2013), and it is also one of the most used modelling languages for EA. We validate our proposed methodology and modelling with the use of a case study.

The research methodology we follow in this study is design science as proposed by Peffers et al. (2007), which has also shaped the structure of this paper. The introduction presents the problem addressed in this paper together with the contribution of the proposed approach. Section 2 includes a presentation of the CBP methodology developed by the authors. In Section 3 we assess the suitability of the ArchiMate core modelling language and its extensions. Section 4 contains a validation of our proposed methodology and metamodel with the help of a case study. The paper concludes with conclusions, discussion, limitations, and future work (Section 5).

\section{CBP METHODOLOGY}

In practice, there are many views on capabilities, mainly because they are being used in many different domains. In the defence industry, a capability refers more to what you would like to be able to do in a specific situation (e.g. war), rather than the ability, capacity or potential that an organisation, person or system possesses (Open Group, 2011) in any type of situation, opportune or not. We will introduce a basic and simple view on capabilities and illustrate this with an example. Simply said, a capability is the ability of an organisation to employ resources to achieve some goal (Iacob et al., 2012). For example, a Customer data management capability is the ability of an organisation to manage the personal information of customers in databases. Thus, we see capabilities as the ways in which enterprises combine resources, competences, information, processes and their environments to deliver value to stakeholders. They describe, in general and high-level terms, what the business is able to do (Open Group, 2011).

The most common use of capabilities is in the context of CBP. According to the Open Group (2011), CBP focuses on the planning, engineering, and delivery of strategic business capabilities to the enterprise. The TTCP is currently using a CBP system that describes generic steps, the input required to be able to do CBP, an assessment tool, and the desired outcome of CBP (Taylor, 2005). However this method is too general and more related to the defence domain. Similarly, the RAND Institute has proposed a method for improving CBP in the American Department of Defence. Although this method is comprehensive, it is intended for military use, therefore it has a focus on risk management and scenario analysis. We have developed our CBP methodology based on the research of Papazoglou (2014), the guidelines of TOGAF (Open Group, 2011), of TTCP (Taylor, 2005) and of the RAND (Davis, 2002).

We identify the following activities in CBP: Map, Assess, and Plan. These activities are typically executed in successive cycles, where some may need more or less attention, depending on what drives the respective planning cycle.

\subsection{Mapping Capabilities}

One of the first steps of CBP is determining the capability map, which contains all the business capabilities of an organisation. The planning of the business improvements should be defined based on this capability map.

\subsubsection{Identifying Capabilities}

Typically, any ability of an organisation can be considered a capability. However capabilities should be defined in a consistent manner. The following guidelines are proposed for doing this. Thus, they

Should be defined by using a language that is understandable by all relevant stakeholders. One of the main benefits of using CBP as a link between Business and IT is that it provides a common language that all stakeholders can understand (van 
Gils and van Dijk, 2014). In order to achieve this, capabilities should be expressed using general and high-level terms (Open Group, 2011). This will ensure that both business leaders and enterprise architects can understand what a capability means in terms of their business.

Should express what the organisation is able to do, not how it does it. According to the Open Group (2011), a capability is the ability that an organisation, person, or system possesses. Therefore it focuses on what an organisation is able to do and abstracts from how the capability is actually achieved (van Gils and van Dijk, 2014). By following this logic, a capability can be defined by using nouns, not verbs (Ulrich and Rosen, 2011).

Should not be redundant. A specific capability should appear only once on a capability map, regardless of how many processes, applications, etc. realise it (Ulrich and Rosen, 2011). This is a consequence of the fact that capabilities should be defined independently of how they are realised.

Should be measureable. Capabilities can be used to guide investment decisions, based on the business outcome(s) they help achieve. Therefore, if the value that is expected to be delivered by improving a capability is not objectively identified, an organisation might make investments that might not yield the expected returns. Thus capabilities should be defined using the SMART guidelines in order to avoid ambiguity (Open Group, 2011)

Can be defined vertically or horizontally. Capabilities can be defined down any lines that an organisation wishes to improve, such as process, function, organisational, etc. Therefore capabilities can become lines of optimisation for an organisation. For example, an organisation that defines capabilities along the lines of processes will optimise their process performance. An organisation that defines capabilities down functional lines will be optimised based on business functions (Open Group, 2011). Consequently it is advised for organisations, depending on their preference, to choose vertical or horizontal optimisation and define their capabilities accordingly.

Can be decomposed into sub-capabilities. It is usual that high-level capabilities are decomposed into more detailed capabilities. This decomposition is particularly useful when making a capability map. For a high-level planning and analysis, such a map may contain main capabilities (level 1) and another two levels of decomposition (level 2 and 3) of these capabilities (Ulrich and Rosen, 2011). It is important to keep the same level of detail for all the capabilities within the same level.

\subsubsection{Linking Capabilities}

As mentioned before, CBP focuses on the planning, engineering, and delivery of strategic business capabilities to the enterprise (Open Group, 2011). There are two main aspects to this statement. First of all, it is stated that CBP deals only with the strategic business capabilities of an organization. What is meant by this is that there should be an emphasis on those capabilities that provide strategic value. This can be done by identifying which capabilities of an organisation contribute to realizing a specific strategy. Therefore the activities of CBP can start in the later phases of strategy planning, after the strategic objectives, KPIs, targets and initiatives have been determined. This strategic guidance is needed to begin CBP (Taylor, 2005).

Second of all, the statement prescribes what CBP should do, namely the planning engineering and delivery of these capabilities, or to put it differently the entire process of obtaining them. It can be further explained as a planning discipline, in which enterprise change is defined, sequenced, coordinated and managed in terms of capability increments. Thus it has impact on and complements EA. This comes in addition to projects and deliverables within the frame of EA, and can, therefore, support project portfolio management as well. In other words, capabilities can be used as a higher level abstraction of EA, where elements of the architecture realise business capabilities. Project portfolio management can help manage those projects that implement enterprise transformation in steps and therefore the realisation of the respective capability increments.

\subsection{Assessing Capabilities}

It is important for CBP to have capabilities that are measurable. Therefore it is necessary to define metrics for each capability. Besides having an objective look at the outcomes of improving a capability, defining metrics can help with assessing the current and desired performance levels, with monitoring progress, and with evaluating the realised outcomes of the improvement (Taylor, 2005). We define a metric as the extent, quantity, amount, or degree of something, as determined by measurement or calculation.

\subsubsection{Identifying Metrics}

In the context of ArchiMate, Metric can be seen as a specialisation of Driver. For example the Process variance metric could be used to measure the level 
of the Customer management capability. In this example, the Process variance metric is a decomposition of the higher level metric Operational performance which is a strategic $\mathrm{KPI} /$ metric.

\subsubsection{Analysing Capability Gaps}

The purpose of this step is to determine the current performance levels of each capability and compare to the desired levels which will help realise the strategic goals. The difference between these current and desired levels is called a capability gap. Once the capability increments have been identified for each business capability, their performance levels can be determined. This is achieved by assessing each capability increment against each metric, typically with a quantitative analysis. In case a quantitative analysis is not possible, a qualitative analysis with pseudo-performance levels can be used. The following is an example of such performance levels: One - Very low; Two - Low; Three - Medium; Four - High; Five - Very high.

This information can be plotted in a spider chart in which capability increments are assessed based on their performance levels according to a several metrics. Any number of metrics can be used to assess the capability increments but a minimum of three metrics are needed to make a spider chart.

The capability heat map can also be used as a high level method for representing capability gaps (Taylor, 2005). After assessing the relevant capabilities in the capability map and determining their desired future levels, a specific colour can be assigned to their performance levels. For example, if a capability is scoring very low on its performance and the desired level is medium, it can be coloured red. If a capability is scoring medium and the desired level is high, then it can be coloured yellow. If a capability is scoring medium and the desired level is medium, then it can be coloured in green.

\subsection{Planning Capability Increments}

The purpose of this section is to plan the improvements to a capability. Typically these types of improvements are not all made at once, but they are planned in capability increments, over time. This implies that the improvement of a capability is usually done in multiple capability increments, each providing a part of the expected added value of improving the capability (Open Group, 2011).

A capability roadmap can be used to sequence and plan these capability increments over time (Papazoglou, 2014). By using this method, it can become easier to have an overview of when each increment is supposed to be implemented. Furthermore it can help with planning the appropriate resources needed to realise each increment and avoid not having resources available because they are being used for other purposes.

In terms of EA, capability roadmaps can be a very useful starting point for planning the necessary architectural change. They can be used to plan the work packages that help realise capability increments. Furthermore they help link desired business value to architectural change and to work packages. Therefore investments in IT can be easier justified as necessary for achieving strategic goals.

\section{MODELLING CBP WITH ARCHIMATE}

Based on the methodology presented in Section 2 we have identified several concepts which we use for determining the suitability of the ArchiMate language for CBP. A complete description of the ArchiMate language can be found in the ArchiMate 2.1 Specification (Open Group, 2013).

- Mission, Vision, Strategy and Objective can be modelled by using the concept of Goal, according to Aldea et al. (2015);

- Analysis/Assessment can be modelled with the concept of Assessment;

- Capability is defined as an organisation's ability to employ resources to achieve some goal. This concept does not have a direct equivalent in ArchiMate and was proposed as an addition by Iacob et al. (2012) and Azevedo et al. (2013);

- Capability Increment is defined as a version of a capability that represents an increase in the performance of the capability. This concept also does not have a direct equivalent in ArchiMate;

- Metric is defined as the extent, quantity, amount, or degree of something, as determined by measurement or calculation. ArchiMate does not contain a concept that can express a measurement assigned to another concept.

From this we can conclude that the current specification of the ArchiMate language is not sufficient for the purposes of CBP. Therefore we propose the addition of the concepts of Capability, Capability increment, and Metric. Figure 1 shows how these concepts can be related to existing ArchiMate concepts. This metamodel, which is based on the work of Iacob et al. (2012) will be used in this paper to model CBP with ArchiMate. 


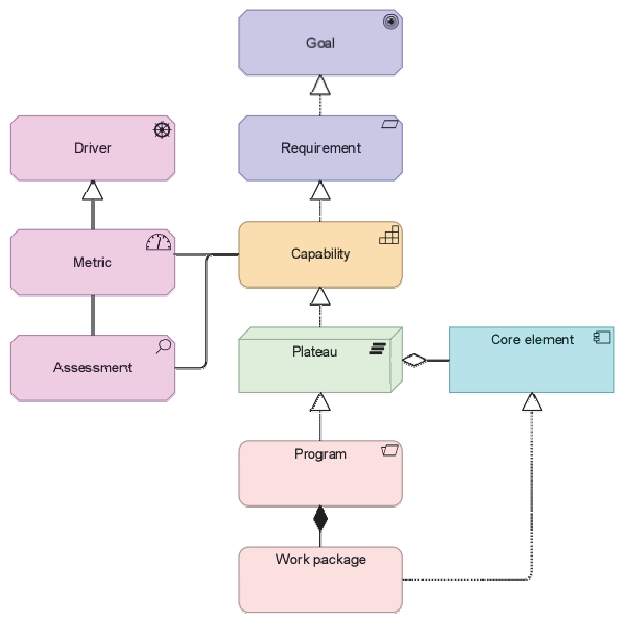

Figure 1: CBP extension metamodel (Iacob et al. 2012).

It is worth noting that Capability increment is not present at metamodel level. This is the case because it is a version/variant of the Capability concept, and not an independent concept within the metamodel. The Capability increment can be modelled as a specialisation of a Capability (at model level), where a specialisation relationship is used to represent that an increment is a version of the Capability (with a certain performance level).

\section{CASE STUDY}

ArchiPharma is a large international organisation that has many geographically spread locations. It is the result of many mergers and take-overs. They are aware of the necessity to continuously change and improve to reach their end goal of becoming the leading provider of pharmaceutical services in the world. To realize this ambitious goal they move their strategy from a complete focus on product leadership to a focus on operational excellence with product leadership still present in the background.

The main issue the organisation is facing is that it needs to comply to many governmental regulations which change regularly. Thus the organisation has to be agile, which is not easy, partly because of the legacy application landscape. The legacy is a result of the many mergers and takeovers where landscapes are simply patched together. These inefficiencies are directly influencing interactions with customers when running daily business. In order to deal with this issues they plan an enormous transformation. Their main concern is how to manage this. In the following sections we show how we supported this transformation by relating disciplines like strategy management, CBP and EA, creating a holistic overview on the transformation. The entire example of the ArchiPharma organisation is modelled using the ArchiMate language (2.1 Specification) and the proposed added concepts of Capability and Metric.

\subsection{Strategy Planning}

Based on their strategy to excel at operations, the organisation has as main objective to Centralise the Information Systems. In order to measure the performance of the Centralize IS objective they use several strategic KPIs such as Process performance, Process variance, and Information consistency.

By assessing how well the objective of Centralize IS can be fulfilled at the moment, we can see there are several problems that stand in the way of successfully realising it. The objective is assessed based on the metrics that are associated with it (Figure 2). It appears that the Process performance metric scores low because there are Multiple and inconsistent CRM databases; Process variance metric scores low because it is Difficult to comply with new regulations due to complex landscape; Information consistency metric scores low because there is a Non-uniform way of billing customers.

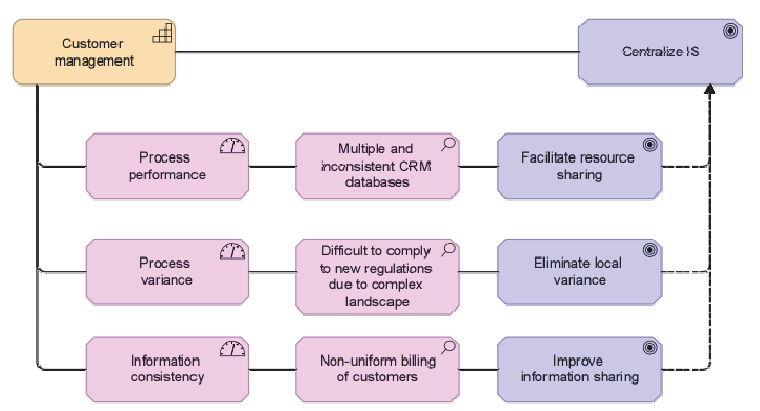

Figure 2: Example objective assessed based on metrics.

\subsection{Capability-based Planning}

In order to proceed with implementing the chosen strategy and objectives, a capability map has been developed. This map contains several main capabilities and their decomposition into subcapabilities. Figure 3 illustrates an excerpt from the capability map of ArchiPharma.

Next, the capabilities which are needed to realise the chosen strategy and the objectives associated to it are identified (Figure 4). These are the strategic capabilities, in the context of the chosen strategy. The underlying architecture which realises each capability is determined. We take the example of the 
Customer management capability.

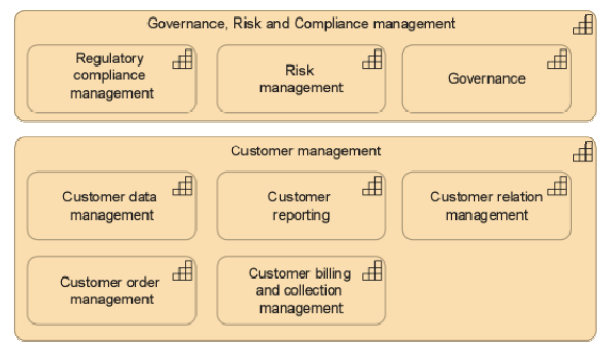

Figure 3: Excerpt of the capability map.

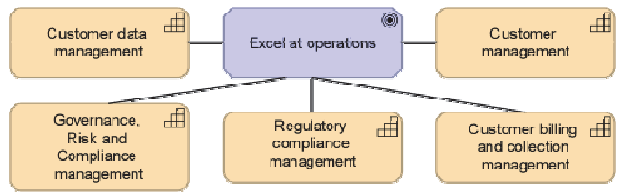

Figure 4: Capabilities related to strategy.

The part of the architecture which is needed to realise this capability is modelled in a plateau (Figure 5).

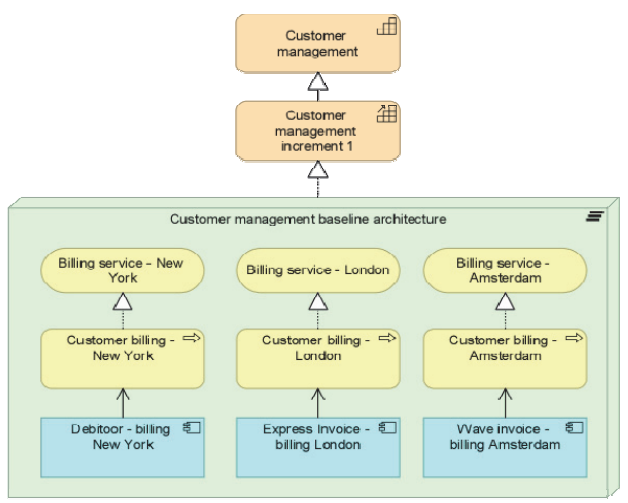

Figure 5: Architecture elements that realise a capability.

We consider that Customer management increment 1 is the current version of the Customer management capability, and the Customer management increment 2 is the next version of the capability, which will help realise the objectives and strategy. With this in mind, increment 1 and 2 are assessed according to the three metrics (Figure 6). Of course the end goal is that the capability will score very high on all metrics, but that is for the following increments (3, $4,5, \ldots, \mathrm{n})$.

Since there are at least three metrics defined for this capability, and thus for its increments, a spider chart can be made. This spider chart shows us in a more graphical way the same information that is available in the capability scorecard (Figure 7).

Based on the assessment of the capabilities we

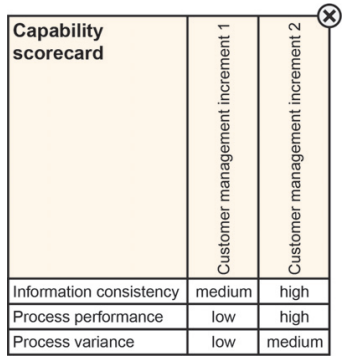

Figure 6: Capability scorecard.

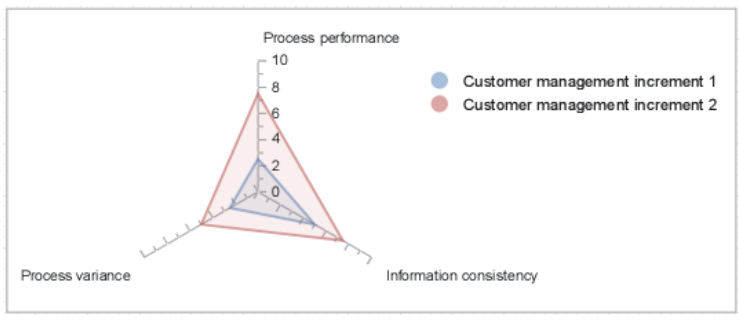

Figure 7: Capability spider chart.

can build a capability heat map. In our example, the Customer management capability can be coloured red since the gap between de current and desired performance levels is fairly large. By looking in depth to its decomposition we can see that also Customer data management and Customer billing and collection management can be coloured red since the gap between the current and desired performance levels is also fairly large (Figure 8).

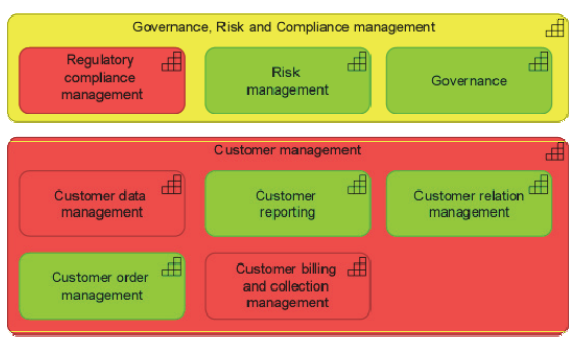

Figure 8: Capability heat map.

Now that the capabilities that need to be improved have been identified, a capability roadmap can be designed. In our example, we plan the improvement of the Customer management and the Governance, Risk and Compliance management capabilities over the next 7 quarters (Figure 9).

\subsection{Enterprise Architecture}

As mentioned before, a capability is realised by core elements of the EA. This can be modelled by using a realisation relation between the capability concept 
and the elements of the ArchiMate core. In our example, we model the architectural elements needed to realise the Customer management capability increment 2, as shown in Figure 10. The plateau concept can be used to organise the transformation planning needed to implement the capability increment.

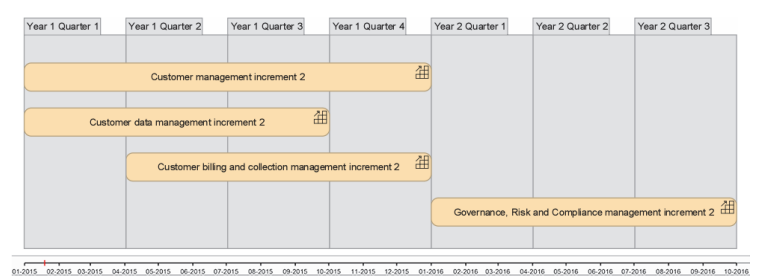

Figure 9: Capability roadmap.

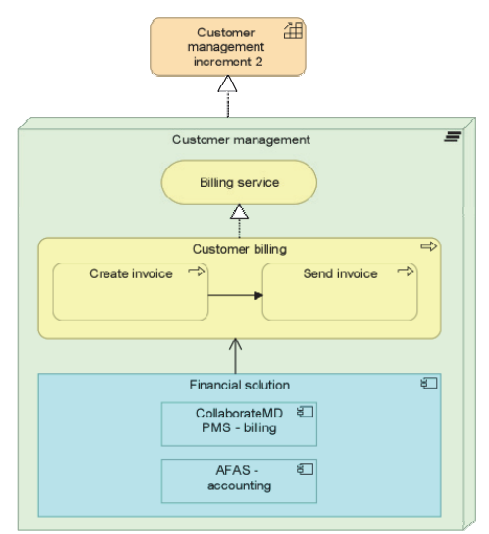

Figure 10: Architecture elements realising a capability increment.

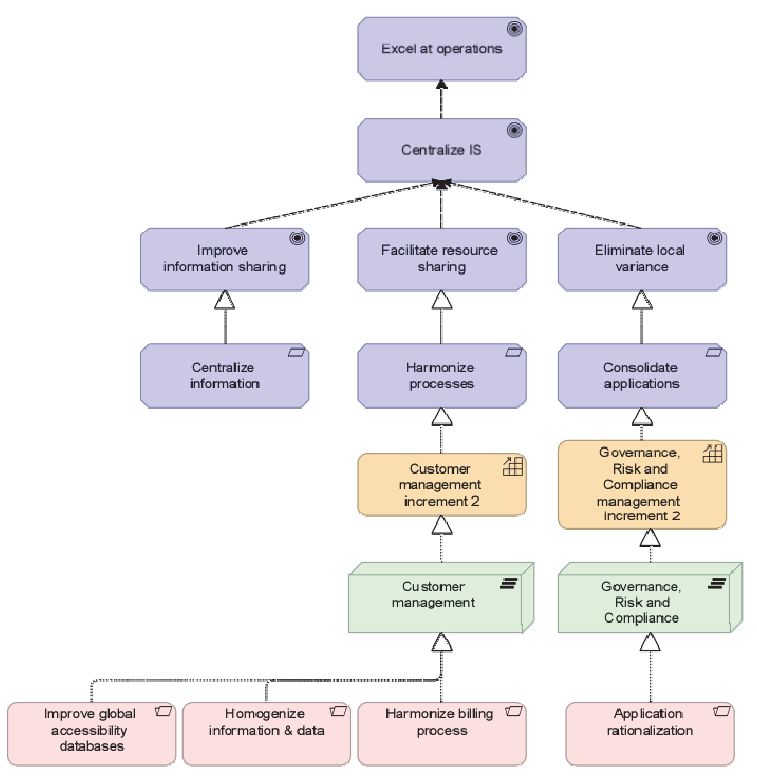

Figure 11: Plateaus that realise capability increments.
For each of the defined plateaus there is at least one program that will realise it. Each of these programmes can have at least one work package (project) that they are composed of.

Figure 11 illustrates such an approach in the context of the ArchiPharma case. By having a relation between work packages/programs and capabilities it is possible to make a roadmap in which it is made clear which work package/program contributes to realising which plateau, which capability increment, and lastly which capability. In our example, we can see in Figure 12 that there are three work packages/programs which help realise the desired version of the Customer management capability. The timeline shows in which period of time these work packages/programs are supposed to be implemented, in which period of time a specific architecture and capability increment will exist.

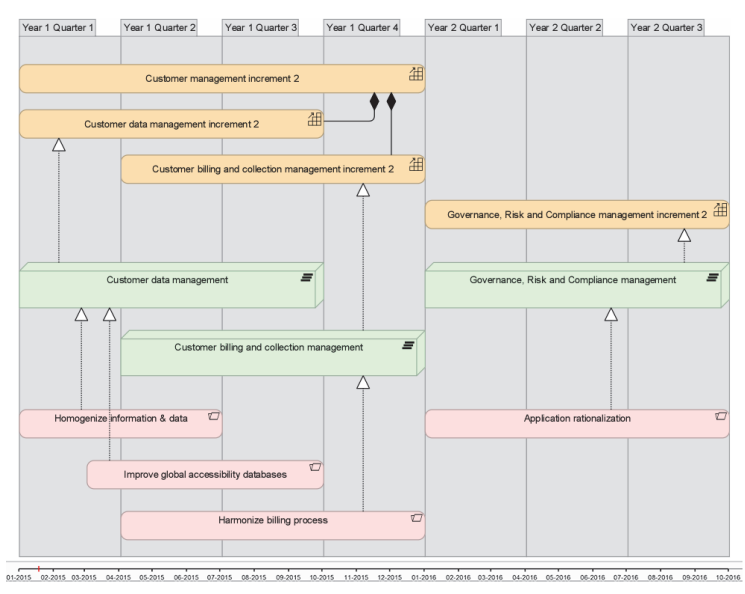

Figure 12: Roadmap with programmes linked to the capabilities they improve.

\section{CONCLUSIONS}

In this paper we propose a methodology for CBP. In order to do this, we look at existing literature and practice, and design methodology that can be used independently, or in combination with strategic management and EA. Based on this methodology we assess the suitability of the ArchiMate modelling language. We show that the language does not include all relevant concepts needed to model CBP. With the addition of the Capability concept (with Capability increment as specialisation) and the Metric concept (as a specialisation of Driver) it is possible to model all aspects of CBP. The first question to answer when considering adding a Capability concept to ArchiMate is: Does ArchiMate 
need a Capability concept? If an existing concept can suffice, then there would be no reason to add another to the language. Following the proposal of Iacob et al. (2012), we argue that a capability is fundamentally different than a business process, business function, business service and business interaction. A capability, as it is also defined in TOGAF, is on a different level of abstraction than the business layer concepts of ArchiMate. Based on this we can state that a capability can be realised by elements of an architecture, such as business process, business function, business service and business interaction.

By being able to use CBP as a link between strategy management and EA it can be possible to achieve a Business and IT alignment. Therefore we suggest that further research should be done in order to investigate this possibility.

There are several limitations to the research we have presented. We have determined that the ArchiMate language is not sufficiently developed at the moment to support CBP modelling. Further research is needed in order to determine if the proposed added concepts are sufficient. Also, we have validated our proposed methodology and metamodel with the help of one case study. Although this is sufficient for stating that our approach is viable for the organisation under analysis, we cannot state that it is applicable for all organisations. Therefore further research needs to be done in order to investigate the generalizability of our methodology and metamodel.

\section{ACKNOWLEDGEMENTS}

The authors thank Bill Poole from Journey One for the work he has done on this topic, which has been an inspiration for this research.

\section{REFERENCES}

Aldea, A., Iacob, M.E., Van Hillegersberg, J., Quartel, D., Franken, H. \& Bodenstaff, L., 2015. Modelling strategy with ArchiMate. In 30th ACM Symposium on Applied Computing ( $S A C$ ). Accepted.

Audia, P.G., Locke, E.A. \& Smith, K.G., 2000. The paradox of success: An archival and a laboratory study of strategic persistence following radical environmental change. Acad. of Man. J., 43, 837-853.

Azevedo, C.L., Iacob, M.E., Almeida, J.P.A., Van Sinderen, M., Pires, L.F. \& Guizzardi, G., 2013. An ontologybased well-founded proposal for modeling resources and capabilities in ArchiMate, In 17th IEEE EDOC, 39-48.
Barney, J. 1991. Firm resources and sustained competitive advantage. Journal of management, 17, 99-120.

Danesh, M.H. \& Yu, E., 2014. Modeling Enterprise Capabilities with $i^{*}$ : Reasoning on Alternatives. Advanced Information Systems Engineering Workshops, 2014, 112-123. Springer.

Davis, P.K., 2002. Analytic architecture for capabilitiesbased planning, mission-system analysis, and transformation. DTIC Document.

De Spiegeleire, S., 2011. Ten trends in capability planning for defence and security.The RUSI Journal, 156,20-28.

Eisenhardt, K.M. \& Martin, J.A., 2000. Dynamic capabilities: what are they? Strategic management journal, 21, 1105-1121.

Hales, D. \& Chouinard, P., 2011. Implementing Capability Based Planning within the Public Safety and Security Sector. DTIC Document.

Iacob, M.E., Quartel, D. \& Jonkers, H., 2012. Capturing business strategy and value in enterprise architecture to support portfolio valuation. In $16^{\text {th }}$ International EDOC, 11-20.

Kraaijenbrink, J., Spender, J.C. \& Groen, A. J., 2010. The resource-based view: a review and assessment of its critiques. Journal of management, 36, 349-372.

Lee, H. \& Song, Y.T., 2011. Bridging Enterprise Architecture Requirements to ArchiMate, Springer.

Miklos, J., 2012. A meta-model for the spatial capability architecture. J. of Theoretical and Applied IT, 43.

Open Group, 2011. TOGAF® Version 9.1, Van Haren.

Open Group, 2013. ArchiMate ${ }^{\circledR} 2.1$, Van Haren.

Papazoglou, A. 2014. Capability-based planning with TOGAF ${ }^{\circledR}$ and ArchiMate ${ }^{\circledR}$. Master thesis, University of Twente.

Peffers, K., Tuunanen,T., Rothenberger,M.A. \& Chatterjee, S., 2007. A design science research methodology for information systems research. $J$. of management information systems, 24, 45-77.

Scott, J., 2009. Business Capability Maps: The Missing Link Between Business Strategy and IT Action. Architecture \& Governance magazine.

Stirna, J., Grabis, J., Henkel, M. \& Zdravkovic, J., 2012. Capability driven development-An approach to support evolving organizations. The Practice of Enterprise Modeling. Springer.

Taylor, B., 2005. Guide to Capability Based Planning. Meeting Proceedings of RTO-MP-SAS-055Analytical Support to Defence Transformation: The RTO Studies, Analysis and Simulation Panel (SAS) Symposium, 26-28 April, 8-1.

Teece, D. \& Pisano, G., 1994. The dynamic capabilities of firms: an introduction. Industrial and corporate change, 3, 537-556.

Ulrich, W. \& Rosen, M., 2011. The Business Capability Map: The" Rosetta Stone" of Business/IT Alignment. Cutter Consortium, Enterprise Architecture, 24.

Van Gils, B. \& Van Dijk, S., 2014. The practice of enterprise architecture: experiences, techniques, and best practices, BiZZdesign Academy B.V.

Zdravkovic, J., Stirna, J., Henkel, M. \& Grabis, J., 2013. Modeling business capabilities and context dependent delivery by cloud services. Advanced Information Systems Engineering. Springer, 369-383. 